\title{
Probability of Alzheimer's disease based on common and rare genetic variants
}

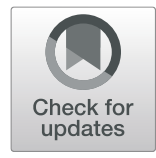

\author{
Valentina Escott-Price ${ }^{1 *}$ (D) and Karl Michael Schmidt ${ }^{2}$
}

\begin{abstract}
Background: Alzheimer's disease, among other neurodegenerative disorders, spans decades in individuals' life and exhibits complex progression, symptoms and pathophysiology. Early diagnosis is essential for disease prevention and therapeutic intervention. Genetics may help identify individuals at high risk. As thousands of genetic variants may contribute to the genetic risk of Alzheimer's disease, the polygenic risk score (PRS) approach has been shown to be useful for disease risk prediction. The APOE- $\varepsilon 4$ allele is a known common variant associated with high risk to $A D$, but also associated with earlier onset. Rare variants usually have higher effect sizes than common ones; their impact may not be well captured by the PRS. Instead of standardised PRS, we propose to calculate the disease probability as a measure of disease risk that allows comparison between individuals.
\end{abstract}

Methods: We estimate AD risk as a probability based on PRS and separately accounting for APOE, AD rare variants and the disease prevalence in age groups. The mathematical framework makes use of genetic variants effect sizes from summary statistics and AD disease prevalence in age groups.

Results: The AD probability varies with respect to age, APOE status and presence of rare variants. In age group 65+, the probability of AD grows from 0.03 to 0.18 (without APOE) and 0.07 to 0.7 (APOE e4e4 carriers) as PRS increases. In 85+, these values are 0.08-0.6 and 0.3-0.85. Presence of rare mutations, e.g. in TREM2, may increase the probability (in 65+) from 0.02 at the negative tail of the PRS to 0.3 .

Conclusions: Our approach accounts for the varying disease prevalence in different genotype and age groups when modelling the APOE and rare genetic variants risk in addition to PRS. This approach has potential for use in a clinical setting and can easily be updated for novel rare variants and for other populations or confounding factors when appropriate genome-wide association data become available.

Keywords: Alzheimer's disease, Disease risk, Polygenic risk score, Rare variants, APOE

\section{Introduction}

Genome-wide association studies (GWAS) identified genetic risk variants of late onset "sporadic" disease beyond the $A P O E$ locus [1-4], followed by exome chip analyses identifying rare variants with moderate risk [5-7]. While causal fully penetrant mutations almost certainly lead to development of the disease [8],

\footnotetext{
* Correspondence: escottpricev@cardiff.ac.uk

1 Dementia Research Institute, Division of Psychological Medicine and Clinical Neurosciences, School of Medicine, Cardiff University, Hadyn Ellis Building, Maindy Rd, Cardiff CF24 4HQ, UK

Full list of author information is available at the end of the article
}

most of the identified singular nucleotide polymorphism (SNP) risk alleles have not been proven to be causal but replicated as carrying an increased disease risk.

The PRSs are designed to aggregate genome-wide genotype data into a single variable indicating genetic liability to a disorder or trait. PRS studies often reach sufficiently high statistical significance to suggest trait polygenicity and, although the prediction accuracy is usually insufficient for clinical utility [9], PRS has been suggested as a useful tool for the selection for clinical trials of individuals of European ancestry across different traits [10-13]. The PRS prediction

(c) The Author(s). 2021 Open Access This article is licensed under a Creative Commons Attribution 4.0 International License, which permits use, sharing, adaptation, distribution and reproduction in any medium or format, as long as you give appropriate credit to the original author(s) and the source, provide a link to the Creative Commons licence, and indicate if changes were made. The images or other third party material in this article are included in the article's Creative Commons licence, unless indicated otherwise in a credit line to the material. If material is not included in the article's Creative Commons licence and your intended use is not permitted by statutory regulation or exceeds the permitted use, you will need to obtain permission directly from the copyright holder. To view a copy of this licence, visit http://creativecommons.org/licenses/by/4.0/. The Creative Commons Public Domain Dedication waiver (http://creativecommons.org/publicdomain/zero/1.0/) applies to the data made available in this article, unless otherwise stated in a credit line to the data. 
accuracy of risk for Alzheimer's disease (AD) is comparatively high, especially when the diagnosis is based upon pathology confirmed rather than clinical assessment (AUC up to 84\%) [14].

Designed to capture the risk of common variants, the PRS aggregates the effects of known genome-wide associated loci [15] and of loci that do not reach genomewide statistical significance. However, the PRS may not well reflect the effect of rare variants in TREM2, PLCG2, $A B I 3, S O R L 1$ [5-7] and very rare highly penetrant mutations in APP, PSEN1 and PSEN2 [16], as the cumulation of many SNPs of small effect sizes tends to mask the strong effect of a single variant. As LD between rare and common variants tends to be small-for example, the maximum $r^{2}$ between a rare variant with minor allele frequency $(\mathrm{MAF})=0.01$ and a typical common SNP with MAF $=0.2$ is $r^{2}=0.04$ when the rare alleles of both variants appear on the same haplotype (i.e. $D^{\prime}=1$ ) [17] - rare variants and PRS are likely to be independent.

Variants in the $A P O E$ gene highly affect the $\mathrm{AD}$ risk $(\mathrm{OR}=3.2, \mathrm{MAF}=0.14)[2] . A P O E$ is also associated with lower odds of reaching the over 90th percentile age [18] as it modifies the age at onset; for example, the age at onset of $\mathrm{AD}$ for $\varepsilon 4 \varepsilon 4$ carriers is $\sim 68$ years [19]. Also, $\varepsilon 4 \varepsilon 4$ carriers are more likely to develop other conditions associated with lower life expectancy such as cardiovascular disease and diabetes [20]. People with AD diagnosed in their late 60s live on average 7 years after the clinical diagnosis, whereas $\mathrm{AD}$ diagnosis after age 90 is associated with an expected survival of only 2.8 years $[21,22]$. Since age is the major confounding factor to the $\mathrm{AD}$ risk, it is difficult to disentangle the ageing and disease pathogenic components.

There is little research on whether APOE and PRS can be modelled as independent variables. Leonenko et al. [23] show that $\varepsilon 4$ frequency decreases with age in both cases and controls, whereas the PRS values are higher in older $\mathrm{AD}$ patients, indicating a negative correlation in cases, but apparently not in controls. These contravariant effects in cases cancel out when a PRS is formed including $A P O E$ alongside other SNPs, so subsequent adjustment for age is ineffective. The use of $A P O E$ genotypes and the PRS (calculated without $A P O E$ ) as two separate predictors accounts for this effect and increases the case/control prediction accuracy but cannot be extended to disease prediction in age groups with different disease prevalence. The approach suggested in the present study accounts both for the age related $A P O E$ effect and different disease prevalence. We propose estimating the disease probability (between 0 and 1 ) based on the PRS while accounting separately for high effect size variants and rare highly penetrant mutations. We show the utility of our probability calculations in application to $\mathrm{AD}$.
Our calculations only require the mean and variance of PRS in cases and in controls and the disease prevalence as reference data, thus avoiding the need to share background sensitive genetic data. While the PRS from different studies (with different SNP selection and/or standardisation) cannot be directly compared, disease probability, as a general quantity, can be used for comparative prioritisation of individuals.

\section{Material and methods PRS distribution}

The PRS aggregates the effects of multiple genetic markers identified by GWAS. Generally, the PRS is expected to be higher in cases than in controls, indicating a higher genetic risk for the disorder, but the difference in mean PRS between case and control samples may be small. It is important to note that the PRS calculated for an individual does not provide an absolute measure of risk and is meaningless except in relation with the distribution of PRS in cases and non-cases in the underlying population.

The polygenic risk score for individual $j \in\left\{1, \ldots, N_{\text {ind }}\right\}$ is $P R S_{j}=\frac{1}{N_{\text {snps }}} \sum_{i=1}^{N_{\text {snps }}} g_{i j} \beta_{i}$, where $N_{\text {ind }}$ and $N_{\text {snps }}$ are the numbers of individuals and of SNPs contributing to the PRS, respectively, $g_{i j} \in\{0,1,2\}$ is the genotype of SNP $i$ for individual $j$, and $\beta_{i}$ is the effect size (logarithm of the odds ratio or logistic regression coefficient) of SNP $i$ in an independent GWAS for the disease. The sample mean and variance are

$$
\begin{aligned}
m(P R S) & =\frac{1}{N_{\text {ind }}} \sum_{j=1}^{N_{\text {ind }}} P R S_{j} \quad \text { and } \\
\operatorname{var}(P R S) & =\frac{1}{N_{\text {ind }}} \sum_{j=1}^{N_{\text {ind }}}\left(P R S_{j}-m(P R S)\right)^{2} .
\end{aligned}
$$

\section{Estimation of PRS distribution parameters for unscreened controls}

Our calculations require the distribution parameters of the PRS in cases and non-cases (putative non-affected controls). If the mean $m_{0}$ and variance $\sigma_{0}^{2}$ of the PRS distribution in non-cases are unknown as unscreened population controls are used, they can be inferred from the means $m_{1}, m_{p}$ and variances $\sigma_{1}^{2}, \sigma_{p}^{2}$ of the PRS distributions in cases and in the population, respectively, and the disease prevalence $K$ as

$$
m_{0}=\frac{m_{p}-K m_{1}}{1-K}
$$

and 


$$
\sigma_{0}^{2}=\frac{\sigma_{p}^{2}-K \sigma_{1}^{2}}{1-K}-\frac{K\left(m_{p}-m_{1}\right)^{2}}{(1-K)^{2}}
$$

(see Supplemental Note 1 for details).

\section{Estimation of the probability of disease development}

By Bayesian inversion, a raw probability $\hat{P}$ to be affected by the disease can be inferred from an individual's PRS value $x$ and the distribution densities of PRS in cases, $p_{1}$, and in controls, $p_{0}$, as

$$
\hat{P}(x)=\frac{K p_{1}(x)}{K p_{1}(x)+(1-K) p_{0}(x)}
$$

However, $\hat{P}$ cannot be directly interpreted as a probability of disease. Logistic regression from case/control samples gives the probability of disease in the logistic model

$$
P(x)=\frac{1}{1+e^{-(\alpha+\beta x)}}
$$

with coefficients $\alpha, \beta$ arising as regression parameters from the maximum likelihood estimate. We use linear regression with the logit link function, taking as data the log odds ratio corresponding to (2),

$$
y=\log \frac{K p_{1}(x)}{(1-K) p_{0}(x)},
$$

at every PRS value $x$ and the joint probability density of PRS in the population, $p_{p}(x)=K p_{1}(x)+(1-K) p_{0}(x)$, as weight. For normal densities $p_{1}$ and $p_{0}$, the coefficients $\alpha, \beta$ can be expressed as

$$
\begin{aligned}
& \alpha=\log \frac{K \sigma_{0}}{(1-K) \sigma_{1}}+\frac{1}{2}\left(\left(r_{0}-1\right) K+\left(1-r_{1}\right)(1-K)\right)-m_{p} \beta, \\
& \beta=\frac{m_{1}-m_{0}}{\sigma_{p}^{2}}\left(K(1-K)\left(\frac{r_{0}+r_{1}}{2}-1\right)+K \frac{\sigma_{1}^{2}}{\sigma_{0}^{2}}+(1-K) \frac{\sigma_{0}^{2}}{\sigma_{1}^{2}}\right)
\end{aligned}
$$

where $r_{1}=\frac{\sigma_{0}^{2}+\left(m_{1}-m_{0}\right)^{2}}{\sigma_{1}^{2}}, r_{0}=\frac{\sigma_{1}^{2}+\left(m_{1}-m_{0}\right)^{2}}{\sigma_{0}^{2}}, m_{p}=K m_{1}+$ $(1-K) m_{0}, \quad$ and $\quad \sigma_{p}^{2}=K \sigma_{1}^{2}+(1-K) \sigma_{0}^{2}+K(1-K)$ $\left(m_{1}-m_{0}\right)^{2}$ (see Supplemental Note 2 for details).

Formulae (5) determine the parameters of the logistic probability model (3) from the disease prevalence and the parameters of the distribution of PRS in cases and non-cases, dispensing with the need to obtain or simulate individual genotypes and perform logistic regression on the resulting PRS. They rely on the assumptions that the PRS distributions are normal and that the raw probability (2) represents well the fraction of cases for any value of PRS. For validation, we compared the outcome of (5) with the following three procedures of increasing abstraction, (a) simulation of genotypes in HWE with given MAF in cases and in non-cases and logistic regression of the resulting PRS, (b) sampling from normal distributions for PRS in cases and in non-cases with parameters $m_{0}, m_{1}, \sigma_{0}^{2}, \sigma_{1}^{2}$ and logistic regression, (c) sampling from the population distribution $p_{p}$ and linear regression of the raw log odds ratio (4).

\section{Inclusion of rare variants in the probability}

The effects of rare genetic variants with high (or medium) disease penetrance may be obscured if modelled as part of PRS including a large number of other SNPs, and the fraction of correctly identified cases carrying a rare mutation will be small in a sample and have little influence on the overall prediction accuracy. Therefore, it seems better to account for them at the level of the disease probability. Suppose we have the logistic regression model for the probability of disease $P_{P R S}$ in terms of the PRS by formulae (3) and (5), excluding the rare variant from the calculation of the PRS. An individual with PRS value $x$ who carries a rare genetic variant with intrinsic probability $p_{\text {rare }}$ to cause the disease has, assuming the effects of the rare variant and of the polygenic risk are independent, the probability of disease

$$
P(x)=P_{P R S}(x)+p_{\text {rare }}\left(1-P_{P R S}(x)\right)=\frac{1+p_{\text {rare }} e^{-(\alpha+\beta x)}}{1+e^{-(\alpha+\beta x)}}
$$

where $x$ is the PRS for the individual. For very rare variant alleles that do not affect the disease prevalence $K$ in the population, the intrinsic probability can be estimated as

$$
p_{\text {rare }}=\frac{K(O R-1)}{K(O R-1)+1},
$$

where $O R$ is the odds ratio (see Supplemental Note 3). The probability $P(x)$ takes values between $p_{\text {rare }}$ and 1 , reflecting the liability of the rare variant to cause the disease even in absence of polygenic risk. In case of several rare variants with mutually independent effect and in-

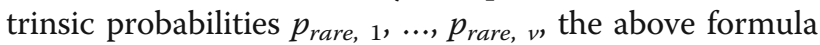
can be applied with $p_{\text {rare }}=1-\prod_{j=1}^{v}\left(1-p_{\text {rare }, j}\right)$. However, due to the assumption of very small allele frequencies, it is unlikely that an individual would carry more than one independent rare variant.

\section{Inclusion of APOE}

It may be advantageous to treat a high-effect common variant such as APOE separately from the PRS. The distributions in cases and non-cases of a PRS formed from SNPs excluding $A P O E$ can be assumed to be approximately equal for carriers and non-carriers of the $A P O E$ 
risk allele. Considering formulae (5), the probability of disease as a function of PRS will then differ between the groups only due to the higher disease prevalence in carriers of the risk allele. Applying (5) with the disease prevalence for the different $A P O E$ genotypes, separate probability curves are obtained. The prevalence in different genotype groups is not usually directly available but can be inferred as follows from the overall prevalence $K$, the overall allele frequency $f$ and the odds ratio $O R$ for the variant, under the assumption of HWE both in the general population and in the subpopulation of noncases. This assumption is justified when the disease prevalence in the population is low (e.g. $2 \%$ for $\mathrm{AD}$ ), but problematic when it is high [24] (e.g. major depression $30 \%)$. The prevalence $K_{0}, K_{1}$ and $K_{2}$ for carriers of nonrisk homozygotes, heterozygotes and risk homozygotes, respectively, can be calculated as

$$
\begin{aligned}
K_{0} & =1-\frac{(1-f-v)^{2}}{(1-K)(1-f)^{2}}, K_{1} \\
& =1-\frac{(1-f-v)(v+f-K)}{(1-K) f(1-f)}, K_{2}=1-\frac{(v+f-K)^{2}}{(1-K) f^{2}},
\end{aligned}
$$

where

$$
v=\frac{b-\sqrt{b^{2}-16(1-O R)(1-f) K}}{4(1-O R)} \text { with } b=2(1+(1-O R)(K-f))
$$

(see Supplemental Note 4).

\section{Standardisation of the probability curve}

PRSs calculated from different sets of SNPs cannot be directly compared. We therefore standardise the PRS axis by expressing the PRS in terms of standard deviations difference from the population mean,

$$
x_{s t}=\frac{x-m_{p}}{\sigma_{p}},
$$

where $x$ is the PRS and $x_{s t}$ is the standardised PRS variable.

\section{Simulated and real data}

Firstly, we simulated independent genotypes in a sample of 10,000 cases and 10,000 controls and used previously published effect sizes for genome-wide significant SNPs $[2,15]$. We calculated an Oligogenic Risk Score (ORS) in the simulated sample using only 39 genome-wide significant SNPs (Supplementary Table 2, adopted from [15]), excluding the APOE proxy SNP (rs429358). The PRS was calculated for 10,039 SNPs, including the above 39 genome-wide significant SNPs and further 10,000 SNPs pruned for LD with $r^{2}=0.1$ and allele frequencies and effect sizes taken from (2).

Secondly, to illustrate the probability of disease in the presence of rare variants, we used effect sizes for rare variants corresponding to the APP, SORL1, TREM2,
$A B I 3$ and PLCG2 genes $[6,7,25]$. We used the distribution parameters $m_{0}, m_{1}$ and $\sigma_{0}^{2}, \sigma_{1}^{2}$ for ORS and PRS as reported in [23] and calculated the disease probabilities with the suggested formulae. To demonstrate the $A P O E$ modelling with our approach, we also took the distribution parameters of $A P O E$, ORS and PRS from the real case/control study [23] (Supplemental Table 1).

The simulations and probability calculations were implemented with R-statistical software. The codes (Simulations.R and Probability.R) can be downloaded from https://github.com/DRI-Cardiff/AD-probability/.

\section{Results}

As the validity of the formulae (5) was established by simulations (see Supplemental Figure 1), we used formulae (3) and (5) to calculate the probability of disease for an individual with PRS value $x$. This probability depends on the disease prevalence in the population of interest, e.g. the general population or a specific subpopulation. The prevalence of $\mathrm{AD}$ in the population depends strongly on age. Recent estimates show a $3 \%, 17 \%$ and $33 \%$ prevalence in the $65-74,75-84$ and $85+$ age groups, respectively [26]. For illustration, we calculated the probability of $\mathrm{AD}$ for $2 \%, 10 \%$ and $30 \%$ prevalence during lifetime and in 65+ and 85+ age groups, respectively. The parameters of the PRS distributions were taken from a real case/control study [23].

Figure 1 shows the dependency of the AD probability $(y$-axis) on standardised PRS ( $x$-axis). The solid thick line corresponds to PRS.AD, calculated as weighted sum of $A P O E$ and PRS.noAPOE with the relative weight of $A P O E$ not taken directly from combining the corresponding effect sizes as a part of PRS but from bivariate logistic regression using APOE and PRS.noAPOE as predictors. The dashed line shows the probability of $\mathrm{AD}$ with PRS calculated in the standard way including all SNPs weighted with their single-SNP effect sizes. In the black and blue scenarios (corresponding to the lifetime and $65+$ prevalences), the standard PRS shows clearer discrimination between low and high probabilities than $A P O E$ alone, somewhat similar to ORS. When the disease prevalence is high (red scenario), then PRS.AD is considerably more discriminative than PRS. This dramatic difference between PRS.AD and PRS is due to the fact that the means and variances for the latter are calculated in cases and controls, ignoring the change in $A P O E-\varepsilon 4$ allele frequency due to age, whereas PRS.AD indirectly accounts for it via the interplay of $A P O E$ and PRS.noAPOE.

Figure 2 demonstrates the results of adding independent rare variant effects in SORL1 and TREM2 to the probability of the disease in both the general population $(K=0.02)$ and in the age group $65+(K=0.1)$. Both 


\section{Probability of Alzheimers disese based on APOE, ORS and PRS}

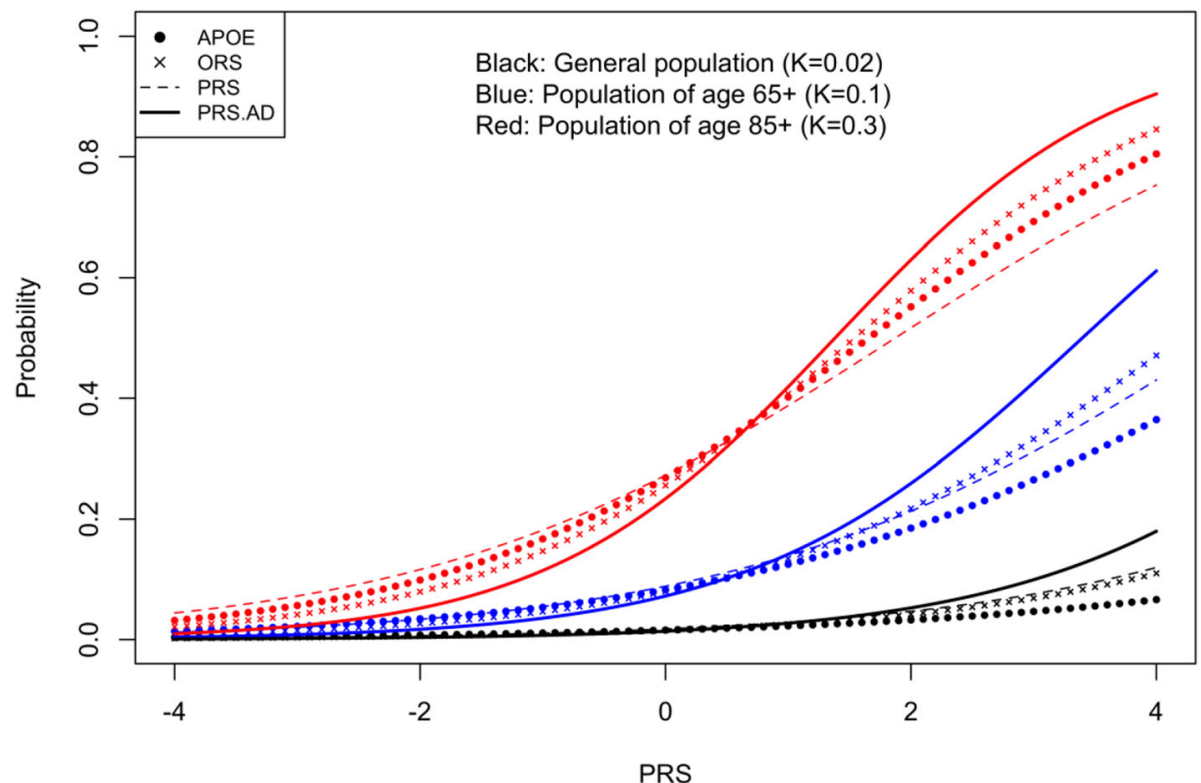

Fig. 1 Probability of Alzheimer's disease, ignoring change in APOE- $\varepsilon 4$ allele frequency due to age. ORS - Oligogenic Risk Score including SNPS with a p-value threshold $p_{T} \leq 10^{-5}$. PRS - Polygenic Risk Score including SNPs with a p-value threshold $p_{T} \leq 0.1$. PRS.AD - PRS calculated as a weighted sum of PRS.no.APOE, including SNPs with a $p_{T} \leq 0.1$ excluding APOE region (CHR19:44.4-46.5), and APOE ( $\left.\varepsilon 2+\varepsilon 4\right)$ ), where APOE effects were weighted with effect sizes $(B(\varepsilon 2)=-0.47$ and $B(\varepsilon 4)=1.12)$ as in Kunkle et al. 2019 [2]

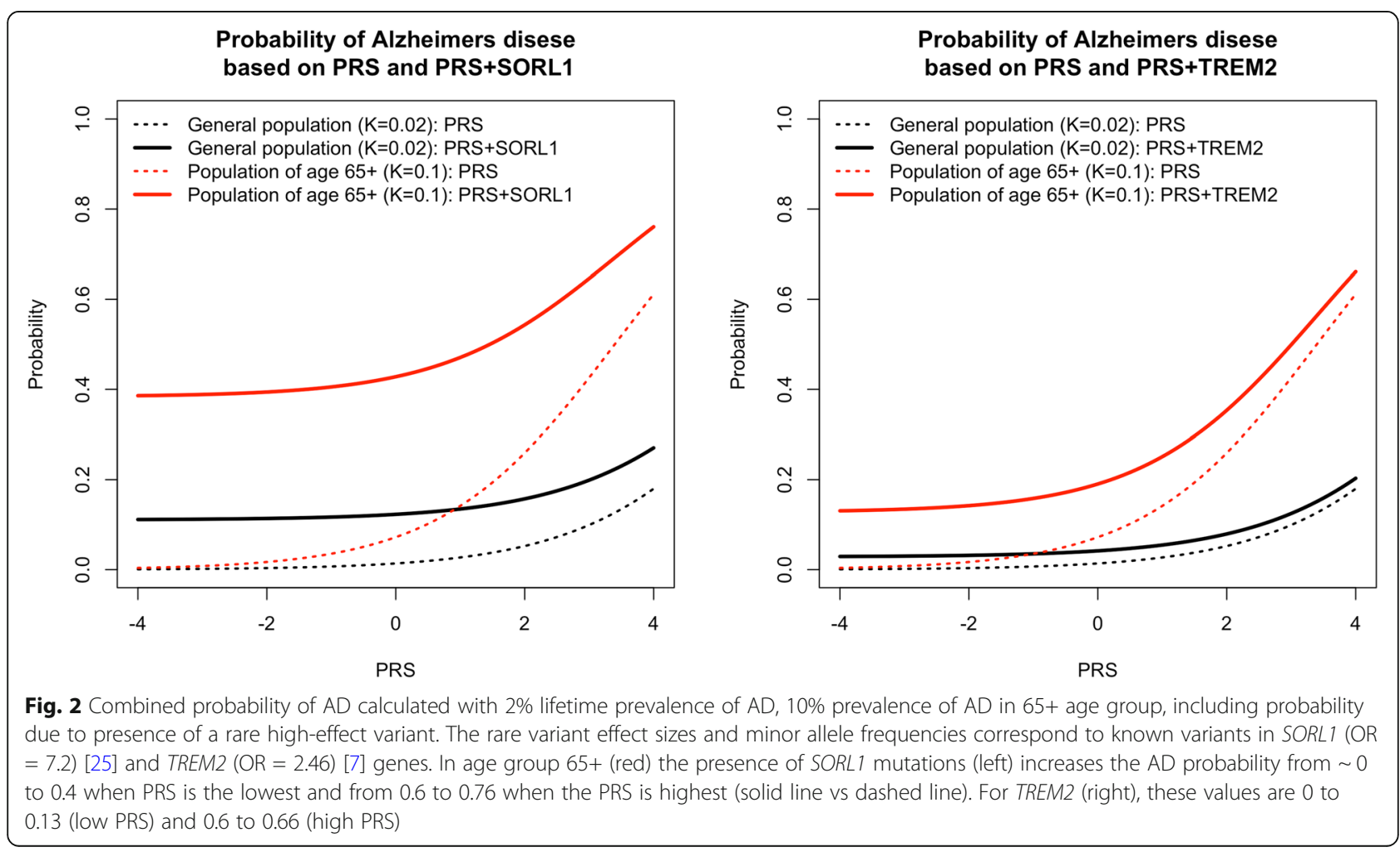


graphs show an elevated disease probability (solid lines), with lower values in the population (black) and higher values in the 65+ group. Dashed lines show the disease probability across the range of PRS if individuals have no rare risk variants. The results for rare variants such as in genes APP, PSEN1 and PSEN2 [27] are shown in Supplemental Figure 2.

Finally, Figure 3 shows the probability of disease in early onset (left) and late onset (right) age groups. As expected, the late onset group shows elevated AD probability even if the PRS is low and APOE $\varepsilon 4$ carriers show consistently higher probability than any other genotypes. The black dashed line shows the disease probability with PRS when the $A P O E$ region is excluded. It is slightly higher than for $A P O E-\varepsilon 4$ non-carriers (thin blue line) as excluding the $A P O E$ region removes the information whether the individuals have lower disease risk due to absence of $\varepsilon 4$ or have protective $\varepsilon 2$ alleles.

\section{Discussion}

PRS do not directly indicate an individual's liability to develop a disease, as they depend on a variety of study parameters such as the number and selection of SNPs included in their calculation and are therefore not comparable between different studies. For comparability, it is not sufficient to standardise the PRS against the population mean and variance; the difference in PRS means in cases and in non-cases is also essential for the interpretation of an individual's PRS. Our proposed calculation of a probability of disease takes all of these properties of the PRS into account and provides a unified measure to assess a PRS value in view of the PRS distribution and disease prevalence in the population or subpopulation of interest. Of course, the choice of SNPs included in a PRS remains decisive for its indicative power.

While the probability curve as a function of standardised PRS can be calculated by logistic regression using genotyped case/control samples from the population of interest, we offer a method to achieve the same outcome using more easily available summary data. The theoretical formula (5) derives the parameters for the logistic probability function (3) from the disease prevalence and the PRS distribution parameters (mean and standard deviation) in cases and non-cases. This conveniently allows estimating an individual's probability of disease from their PRS value using only a small set of parameters. We have shown that (5) gives a highly accurate proxy for case-control sampling of PRS and logistic regression if either the PRS aggregates a high number of SNPs or very highly associated SNPs (such as $A P O E$ variants for $\mathrm{AD}$ ) are excluded from the PRS. If the PRS is calculated from a small number of SNPs including some that are highly associated, deviation from normality in the PRS may lead to some discrepancy, but the formula still gives a close approximation.

Moreover, the probability of disease framework allows for separate consideration of high-effect variants. It has been observed that inclusion of high-effect variants in

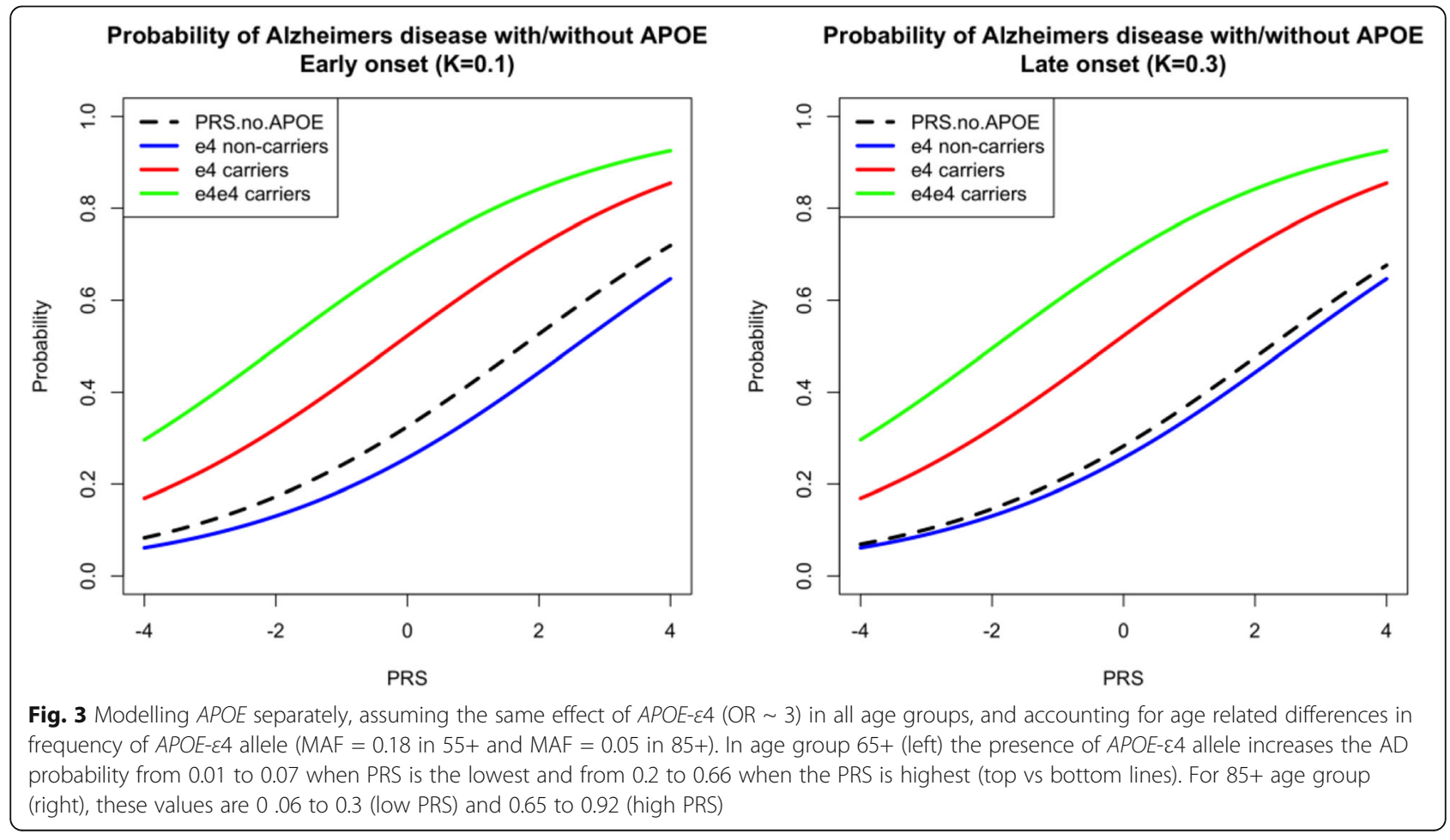


the PRS calculation does not always give optimal results. The impact of common high-effect variants such as $A P O E$ for $\mathrm{AD}$ can vary due to confounders such as age stratification, so taking a summary effect size from a reference study may result in a suboptimal PRS; this becomes apparent when APOE and PRS are used as separate predictors in bivariate logistic regression. We propose including high-effect variants in two different ways in the calculation of disease probability.

The effect of rare, highly penetrant genetic variants tends to be masked by more common variants in the PRS. However, if they do not affect the disease prevalence in the population and act independently of the PRS, we can account for them directly as adding a certain intrinsic probability for carriers of the risk allele. The probability curve approaches this intrinsic probability for highly negative values of standardised PRS.

Common high-effect variants such as $A P O E$ for $\mathrm{AD}$ strongly affect the disease prevalence in the population and cannot be dealt with as above. Although $A P O E$ may not act independently from other genetic causal variants, a recent study [28] suggests that it is reasonable to assume that the distribution parameters for the PRS calculated without $A P O E$ are independent of the $A P O E$ genotype. We propose calculating separate probability curves for each $A P O E$ status, based on the disease prevalence calculated for each $A P O E$ status from the disease prevalence in the population, the risk allele frequency and the odds ratio. These data are available and can easily be further stratified into relevant subpopulations, e.g. by age for $\mathrm{AD}$.

The disease probability allows comparison of PRS calculated from summary data of different reference studies and thus has the potential to be used in a clinical context to prioritise individuals for diagnostics and preventative intervention based on assessed risk of developing the disease.

There are other factors that can influence the disease development probability. For example, it has been reported that $\mathrm{AD}$ is more prevalent in women and PRS effects may also depend on gender [29]. Due to the lack of information on gender-interactions for genetic variants, incorporating gender information in the probability calculations may not be straightforward at present. However, in view of the emerging literature it is likely that this information will be available and reliable in future. It can then be incorporated in our calculations, e.g. by selecting the SNPs for the PRS in males and females separately and/or changing the disease prevalence not by age only, but by sex as well. It is possible to include other, nongenetic predictors. This can be achieved by adjusting the disease prevalence in different e.g. educational attainment groups.
Our approach can be used for other complex genetic disorders. For example, schizophrenia is a highly polygenic disorder [30] and has an increased burden of rare variants and CNVs [31]. It is, however, a neurodevelopmental disorder, and the disease prevalence does not depend on age. It also does not have strong genetic risk factors like $A P O E$ for $\mathrm{AD}$. Therefore, the most relevant probability calculation approach for diseases like schizophrenia and depression is a combination of common and rare variants, while the method shown above for the inclusion of $A P O E$-like variants is not required.

The proposed method relies on the availability of allele frequency and genetic effect size estimates derived from a representative reference population. Expanding this approach to other populations will be possible when the effect sizes of SNPs in other populations will be reliably identified and reported. They can then be used to estimate the disease probability in the relevant population. As in other complex genetic disorders, the disease risk estimates rely heavily on the individual SNP risk estimates and disease prevalence, which differ depending on the demographics, ethnicity and age groups.

\section{Limitation}

A limitation of the present study is that it is based on sound, but theoretical principles, uses SNP and PRS characteristics from the literature and employs simulated data for validation. It remains to test and validate the theory directly in real datasets. Since we are dealing with rare variants, validation of this approach in real data with a sufficient level of confidence will require large population datasets like e.g. the UK BioBank [32]. However, the UK Biobank is not directly suitable to study neurodegenerative disorders, as the cohort is relatively young and only a small proportion of individuals manifest the disease. In addition, it does not provide phenotypic variables which are used to assess cognitive decline in dementia in clinical settings. Publicly available ADspecific datasets are typically small and not suitable to extract a reliable number of people carrying rare mutations (e.g. Alzheimer's Disease Neuroimaging Initiative (ADNI) database (www.loni.ucla.edu/ADNI)). Nevertheless, the approach presented here can be used in small studies focusing on rare and common genetic variants, for example to identify individuals most at risk of developing the disease. It can easily and flexibly be updated as novel rare variants are discovered and as appropriate GWAS data become available for specific populations.

\section{Conclusions}

The proposed method gives an estimate of the probability of developing AD based on an individual's PRS, $A P O E$ genotype and the presence or absence of rare genetic variants associated with $\mathrm{AD}$. The computational 
framework uses as reference data the means and standard deviations of the PRS employed in cases and in (either screened or population) controls and the disease prevalence. The disease prevalence varies considerably in different age and $A P O E$ genotype groups, and the present method allows for taking these differences into account in a natural and transparent way.

\section{Supplementary Information}

The online version contains supplementary material available at https:/doi. org/10.1186/s13195-021-00884-7.

\begin{abstract}
Additional file 1 Supplemental Note 1 Estimate of distribution parameters with unscreened controls. Supplemental Note 2 Estimate of probability of disease by linear regression with logistic link function. Supplemental Note 3 Inclusion of rare variants in the probability. Supplemental Note 4 Inclusion of common variants of common with high effect. Supplemental Table 1 Mean and variance for the Alzheimer's disease genetic risk scores. Supplementary Table 2 AD genome-wide significant SNPs (adopted from Andrewes et al 2020). Supplemental Figure 1 The comparison of the theoretical probability of disease using formulae (3). (5) with the probability of disease estimated by logistic regression of simulated genotypes. Supplemental Figure 2 Combined probability of AD calculated with $2 \%$ lifetime prevalence of $A D, 10 \%$ prevalence of $A D$ in $65+$ age group, including probability due to presence of a rare variant. The effect size is set to $O R=500$ reflecting almost fully penetrant mutations in APP, PSEN1, and PSEN2 genes.
\end{abstract}

\section{Acknowledgements}

Not applicable

\section{Authors' contributions}

KMS and VEP wrote the manuscript and prepared figures and tables. All authors reviewed and approved the final version of the manuscript.

\section{Funding}

Dementia Research Institute (UKDRI supported by the Medical Research Council (UKDRI-3003), Alzheimer's Research UK, and Alzheimer's Society), Joint Programming for Neurodegeneration (JPND, MRC: MR/T04604X/1), DPUK (MR/L023784/2), MRC Centre for Neuropsychiatric Genetics and Genomics (MR/L010305/1).

\section{Availability of data and materials}

The relevant data and materials are available in the supplemental material.

\section{Declarations}

Ethical approval and consent to participate

Not applicable

\section{Consent for publication}

Not applicable

\section{Competing interests}

The authors declare that they have no competing interests.

\section{Author details}

${ }^{1}$ Dementia Research Institute, Division of Psychological Medicine and Clinical Neurosciences, School of Medicine, Cardiff University, Hadyn Ellis Building, Maindy Rd, Cardiff CF24 4HQ, UK. ${ }^{2}$ School of Mathematics, Cardiff University, Senghennydd Road, Cardiff CF24 4AG, UK.
Received: 23 April 2021 Accepted: 5 August 2021

Published online: 17 August 2021

\section{References}

1. Jansen IE, Savage JE, Watanabe K, Bryois J, Williams DM, Steinberg S, et al. Genome-wide meta-analysis identifies new loci and functional pathways influencing Alzheimer's disease risk. Nat Genet. 2019;51(3):404-13. https:// doi.org/10.1038/s41588-018-0311-9.

2. Kunkle BW, Grenier-Boley B, Sims R, Bis JC, Damotte V, Naj AC, et al. Genetic meta-analysis of diagnosed Alzheimer's disease identifies new risk loci and implicates Abeta, tau, immunity and lipid processing. Nat Genet. 2019;51(3): 414-30. https://doi.org/10.1038/s41588-019-0358-2.

3. Lambert JC, Ibrahim-Verbaas CA, Harold D, Naj AC, Sims R, Bellenguez C, et al. Meta-analysis of 74,046 individuals identifies 11 new susceptibility loci for Alzheimer's disease. Nat Genet. 2013;45(12):1452-8. https://doi.org/10.1 038/ng.2802.

4. Marioni RE, Harris SE, Zhang Q, McRae AF, Hagenaars SP, Hill WD, et al. GWAS on family history of Alzheimer's disease. Transl Psychiatry. 2018;8(1): 99. https://doi.org/10.1038/s41398-018-0150-6.

5. Guerreiro R, Wojtas A, Bras J, Carrasquillo M, Rogaeva E, Majounie E, et al. TREM2 Variants in Alzheimer's Disease. N Engl J Med. 2013;368(2):117-27.

6. Jonsson T, Stefansson H, Steinberg S, Jonsdottir I, Jonsson PV, Snaedal J, et al. Variant of TREM2 associated with the risk of Alzheimer's disease. N Engl J Med. 2013;368(2):107-16. https://doi.org/10.1056/NEJMoa1211103.

7. Sims R, van der Lee SJ, Naj AC, Bellenguez C, Badarinarayan N, Jakobsdottir , et al. Rare coding variants in PLCG2, ABI3, and TREM2 implicate microglial-mediated innate immunity in Alzheimer's disease. Nat Genet. 2017:49(9):1373-84. https://doi.org/10.1038/ng.3916.

8. Tanzi RE, Bertram L. Twenty years of the Alzheimer's disease amyloid hypothesis: a genetic perspective. Cell. 2005;120(4):545-55. https://doi.org/1 0.1016/j.cell.2005.02.008

9. Lewis $C M$, Vassos E. Prospects for using risk scores in polygenic medicine. Genome Med. 2017;9(1):96. https://doi.org/10.1186/s13073-017-0489-y.

10. Agerbo E, Sullivan PF, Vilhjalmsson BJ, Pedersen CB, Mors O, Borglum AD, et al. Polygenic Risk Score, Parental Socioeconomic Status, Family History of Psychiatric Disorders, and the Risk for Schizophrenia: A Danish PopulationBased Study and Meta-analysis. JAMA Psychiatry. 2015;72(7):635-41. https:// doi.org/10.1001/jamapsychiatry.2015.0346.

11. Escott-Price V, Shoai M, Pither R, Williams J, Hardy J. Polygenic score prediction captures nearly all common genetic risk for Alzheimer's disease. Neurobiol Aging. 2017:49:214 e7- e11.

12. Lall K, Magi R, Morris A, Metspalu A, Fischer K. Personalized risk prediction for type 2 diabetes: the potential of genetic risk scores. Genet Med. 2017; 19(3):322-9. https://doi.org/10.1038/gim.2016.103.

13. Natarajan P, Young R, Stitziel NO, Padmanabhan S, Baber U, Mehran R, et al. Polygenic Risk Score Identifies Subgroup With Higher Burden of Atherosclerosis and Greater Relative Benefit From Statin Therapy in the Primary Prevention Setting. Circulation. 2017;135(22):2091-101. https://doi. org/10.1161/CIRCULATIONAHA.116.024436.

14. Escott-Price V, Myers AJ, Huentelman M, Hardy J. Polygenic risk score analysis of pathologically confirmed Alzheimer disease. Ann Neurol. 2017; 82(2):311-4. https://doi.org/10.1002/ana.24999.

15. Andrews SJ, Fulton-Howard B, Goate A. Interpretation of risk loci from genome-wide association studies of Alzheimer's disease. Lancet Neurol, 2020;19(4):326-35. https://doi.org/10.1016/S1474-4422(19)30435-1.

16. Tandon A, Fraser P. The presenilins. Genome Biol. 2002;3(11):reviews3014.

17. Slatkin M. Linkage disequilibrium--understanding the evolutionary past and mapping the medical future. Nat Rev Genet. 2008;9(6):477-85. https://doi. org/10.1038/nrg2361.

18. Deelen J, Evans DS, Arking DE, Tesi N, Nygaard M, Liu X, et al. A metaanalysis of genome-wide association studies identifies multiple longevity genes. Nat Commun. 2019;10(1):3669. https://doi.org/10.1038/s41467-019-11 558-2.

19. Liu CC, Liu CC, Kanekiyo T, Xu H, Bu G. Apolipoprotein E and Alzheimer disease: risk, mechanisms and therapy. Nat Rev Neurol. 2013:9(2):106-18. https://doi.org/10.1038/nrneurol.2012.263.

20. El-Lebedy D, Raslan HM, Mohammed AM. Apolipoprotein E gene polymorphism and risk of type 2 diabetes and cardiovascular disease. Cardiovasc Diabetol. 2016;15(1):12. https://doi.org/10.1186/s12933-016-032 9-1. 
21. Garre-Olmo J, Ponjoan A, Inoriza JM, Blanch J, Sanchez-Perez I, Cubi R, et al. Survival, effect measures, and impact numbers after dementia diagnosis: a matched cohort study. Clin Epidemiol. 2019;11:525-42. https://doi.org/1 $0.2147 /$ CLEP.S213228.

22. Tom SE, Hubbard RA, Crane PK, Haneuse SJ, Bowen J, McCormick WC, et al. Characterization of dementia and Alzheimer's disease in an older population: updated incidence and life expectancy with and without dementia. Am J Public Health. 2015;105(2):408-13. https://doi.org/10.2105/A JPH.2014.301935.

23. Leonenko G, Baker E, Stevenson-Hoare J, Sierksma A, Fiers M, Williams J, et al. Identifying individuals with high risk of Alzheimer's disease using polygenic risk scores. Nat Commun. 2021;12:4506.

24. Wang J, Shete S. Using both cases and controls for testing hardy-weinberg proportions in a genetic association study. Hum Hered. 2010;69(3):212-8. https://doi.org/10.1159/000289597.

25. Bellenguez C, Charbonnier C, Grenier-Boley B, Quenez O, Le Guennec K, Nicolas $\mathrm{G}$, et al. Contribution to Alzheimer's disease risk of rare variants in TREM2, SORL1, and ABCA7 in 1779 cases and 1273 controls. Neurobiol Aging. 2017:59:220 e1- e9.

26. Hebert LE, Weuve J, Scherr PA, Evans DA. Alzheimer disease in the United States (2010-2050) estimated using the 2010 census. Neurology. 2013;80(19): 1778-83. https://doi.org/10.1212/WNL.0b013e31828726f5.

27. Van Cauwenberghe C, Van Broeckhoven C, Sleegers K. The genetic landscape of Alzheimer disease: clinical implications and perspectives. Genet Med. 2016;18(5):421-30. https://doi.org/10.1038/gim.2015.117.

28. Bakulski KM, Vadari HS, Faul JD, Heeringa SG, Kardia SLR, Langa KM, et al. Cumulative Genetic Risk and APOE epsilon4 Are Independently Associated With Dementia Status in a Multiethnic, Population-Based Cohort. Neurol Genet. 2021;7(2):e576. https://doi.org/10.1212/NXG.0000000000000576.

29. Fan CC, Banks SJ, Thompson WK, Chen CH, McEvoy LK, Tan CH, et al. Sexdependent autosomal effects on clinical progression of Alzheimer's disease. Brain. 2020;143(7):2272-80. https://doi.org/10.1093/brain/awaa164.

30. Pardinas AF, Holmans P, Pocklington AJ, Escott-Price V, Ripke S, Carrera N, et al. Common schizophrenia alleles are enriched in mutation-intolerant genes and in regions under strong background selection. Nat Genet. 2018; 50(3):381-9. https://doi.org/10.1038/s41588-018-0059-2.

31. Rees E, O'Donovan MC, Owen MJ. Genetics of schizophrenia. Curr Opin Behav Sci. 2015;2:8-14. https://doi.org/10.1016/j.cobeha.2014.07.001.

32. Sudlow C, Gallacher J, Allen N, Beral V, Burton P, Danesh J, et al. UK biobank: an open access resource for identifying the causes of a wide range of complex diseases of middle and old age. PLoS Med. 2015;12(3):e1001779. https://doi.org/10.1371/journal.pmed.1001779.

\section{Publisher's Note}

Springer Nature remains neutral with regard to jurisdictional claims in published maps and institutional affiliations.

Ready to submit your research? Choose BMC and benefit from:

- fast, convenient online submission

- thorough peer review by experienced researchers in your field

- rapid publication on acceptance

- support for research data, including large and complex data types

- gold Open Access which fosters wider collaboration and increased citations

- maximum visibility for your research: over $100 \mathrm{M}$ website views per year

At $\mathrm{BMC}$, research is always in progress.

Learn more biomedcentral.com/submissions 